EPJ Web of Conferences 64, 07003 (2014)

DOI: $10.1051 /$ epjconf/ 20146407003

(C) Owned by the authors, published by EDP Sciences, 2014

\title{
The Peculiar Binary System AE Aquarii from its Characteristic Multi-wavelength Emission
}

\author{
B. Oruru ${ }^{1, a}$ and P. J. Meintjes ${ }^{2, b}$ \\ ${ }^{1}$ Department of Physics, Busitema University, P. O. Box 236, Tororo, Uganda \\ ${ }^{2}$ Department of Physics, University of the Free State, Bloemfontein 9300, South Africa
}

\begin{abstract}
The multi-wavelength properties of the novalike variable system AE Aquarii are discussed in terms of the interaction between the accretion inflow from a late-type main sequence star and the magnetosphere of a fast rotating white dwarf. This results in an efficient magnetospheric propeller process and particle acceleration. The spin-down of the white dwarf at a period rate of $5.64 \times 10^{-14} \mathrm{~s} \mathrm{~s}^{-1}$ results in a huge spin-down luminosity of $L_{\mathrm{s}-\mathrm{d}} \simeq 6 \times 10^{33} \mathrm{erg} \mathrm{s}^{-1}$. Hence, the observed non-thermal hard X-ray emission and VHE and $\mathrm{TeV}$ gamma-ray emission may suggest that $\mathrm{AE}$ Aquarii can be placed in the category of spin-powered pulsars. Besides, observed hard X-ray luminosity of $L_{\mathrm{X} \text {,hard }} \leq 5 \times 10^{30}$ $\mathrm{erg} \mathrm{s}^{-1}$ constitutes $0.1 \%$ of the total spin-down luminosity of the white dwarf. This paper will discuss some recent theoretical studies and data analysis of the system.
\end{abstract}

\section{Introduction}

The novalike variable AE Aquarii (AE Aqr) is a low mass, non-eclipsing close binary system. It consists of a fast rotating magnetized white dwarf $\left(P_{*}=33 \mathrm{~s}, B_{*} \sim 10^{6} \mathrm{G}\right)$ orbiting a late-type secondary star $[8,16]$ with orbital period of $9.88 \mathrm{~h}[32,33]$. The secondary star is a spectral type K3-5 red dwarf star filling its Roche lobe and hence transfers matter to the primary companion [14, 16, 34]. Most cataclysmic variables (CVs) have M-type dwarf secondaries, indicating that the K-type secondary star in AE Aqr suggests a different evolutionary path, perhaps just evolved from the common envelope phase [28].

AE Aqr has been observed and studied across the electromagnetic spectrum, from radio (through optical) to TeV gamma-rays [20]. It was first observed in the optical [37] and later in other wavelengths $[4,5,22,26]$. The most unique characteristic of AE Aqr is perhaps its rapid flaring in almost all wavelengths [15]. Large optical flares and flickering [25], large radio flares [3] and $\mathrm{TeV} \gamma$-ray emission [21] make the system enigmatic [16]. The characteristics of the observed radio flares, which are reportedly distinct from optical, UV, and X-ray flares [1] are associated with transient non-thermal emission processes resembling Cyg X-3 in a high state [4].

AE Aqr reveals itself as a powerful non-thermal variable source in radio and possibly $\mathrm{TeV} \gamma$-rays, Figure $1[2,3,9,21]$, resembling the microquasar Cyg X-3 rather than any of the presently known CVs [14]. A similar radio brightness has never been observed in any of the other CVs. The observed

\footnotetext{
a e-mail: bc.oruru@gmail.com

be-mail: MeintjPJ@sci.ufs.ac.za
}

This is an Open Access article distributed under the terms of the Creative Commons Attribution License 2.0, which permits unrestricted use, distribution, and reproduction in any medium, provided the original work is properly cited. 

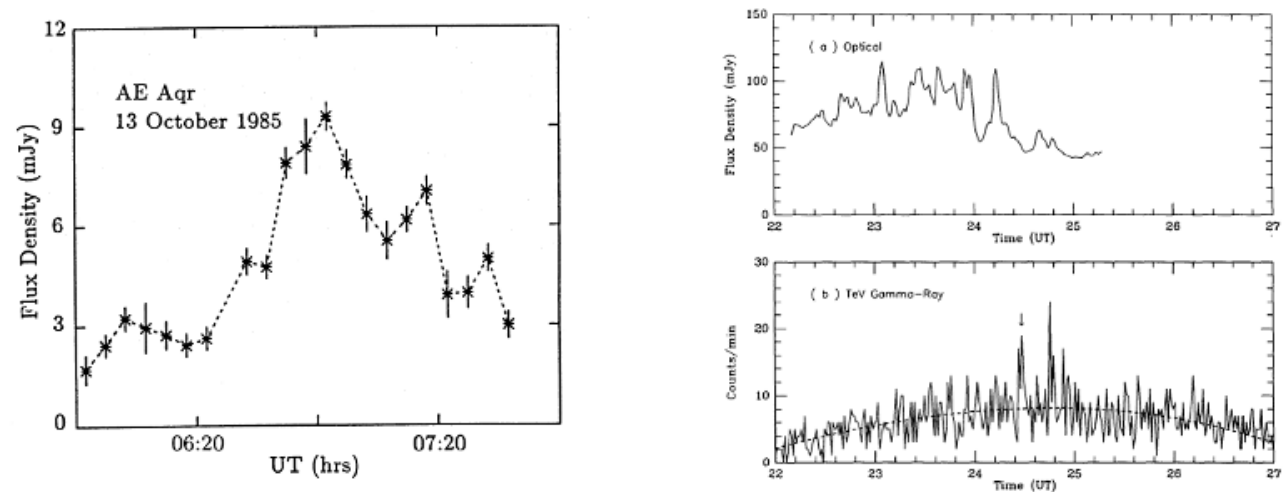

Figure 1. Left: Radio light curve of AE Aqr from VLA data [3]. Right: Optical and $\mathrm{TeV} \gamma$-ray light curves from data observed with the SAAO 30 inch Cassegrain telescope and the Nooitgedacht Mk I Cherenkov telescope [21].

luminosity of the strongest $\mathrm{TeV}$ flare is $\sim 10^{34} \mathrm{erg} \mathrm{s}^{-1}$ corresponds to the inferred spin-down power of the white dwarf $[21,22]$. In the remaining parts of the spectrum, the emission is predominantly thermal. The UV spectrum of AE Aqr shows strong emission lines, which is characteristic of emission from hot optically thin plasma [10]. A recent Suzaku detection has, however, reported non-thermal hard X-ray emission from AE Aqr above $10 \mathrm{keV}$ [29], a component of this paper.

\section{Propeller Spin-down of the White Dwarf}

Using data set spanning $\sim 14$ years, it has been shown that the white dwarf is spinning down at a rate of $\dot{P} \sim 5.64 \times 10^{-14} \mathrm{~s} \mathrm{~s}^{-1}$ [9], which translates to a luminosity of $L_{\mathrm{S}-\mathrm{d}} \simeq 6 \times 10^{33} \mathrm{erg} \mathrm{s}^{-1}$ [24]. A follow-up study revealed a spin-down rate which is $\sim 3.5 \%$ higher [18]. The spin-down luminosity exceeds the UV \& X-ray luminosities, $L_{\mathrm{UV}}=L_{\mathrm{X}} \sim 10^{31} \mathrm{erg} \mathrm{s}^{-1}[10,27]$, by more that two orders.

Although AE Aqr is classified as a DQ Her system [20], its relatively low X-ray luminosity [8], and the structure of the $\mathrm{H}_{\alpha}$ Doppler tomogram [36] argue against the possibility of mass accretion onto the surface of the white dwarf. A proposed model has suggested that most of the accretion outflow is ejected from the system by the fast rotating magnetosphere of the WD, the so-called propeller process $[35,36]$. In this model, the mass ejection takes place before the gas can penetrate into the magnetosphere deeper than the magnetospheric (Alfvén) radius, i.e. where the magnetospheric pressure starts to dominate the gas ram pressure. The flow pattern is depicted in Figure 2. At the radius of closest approach, most of the material of the stream is ejected, with escape velocity of $v_{\text {esc }} \sim 1500 \mathrm{~km}$ $\mathrm{s}^{-1}[23,30]$. As the blobs gain orbital angular momentum, the WD spins down.

\section{Observations of AE Aquarii with Chandra and Swift}

The Chandra X-ray Observatory [6, 31] and the Swift gamma-ray burst explorer [13] observed AE Aqr almost simultaneously [24]. Chandra observed this peculiar source on August 30, 2005 for $\sim 80$ ks $[18,19,24]$. Swift observed it between August 30 and September 2, 2005 for a total duration of $\sim 10.5 \mathrm{ks}$, as a pre-planned target or a target of opportunity $[12,24]$. Processed data was acquired through the HEASARC on-line service, provided by the NASA's GSFC [24]. 


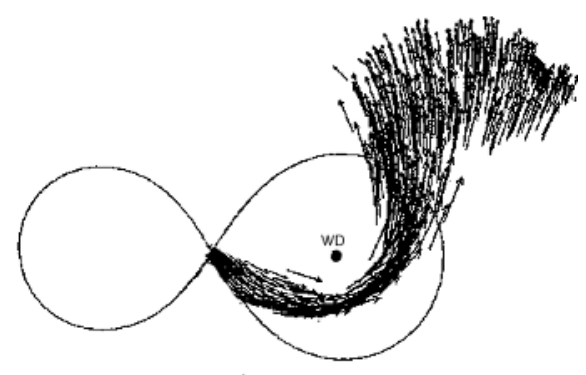

Figure 2. The magnetic propeller in AE Aqr. Distance of closest approach of the stream is $\sim 10^{10} \mathrm{~cm}[36]$.

\subsection{Data Reduction and Analysis}

For both Chandra and Swift XRT data, use was made of the level 2 event files [24]. Data reduction and analysis were done using ciao version 4.2 and heasoft version 6.11 respectively. For light curves, barycentric corrections were applied to the level 2 files by running the axbary and barycorr tools [24]. These were then used to search for periodicities in the data. The Chandra spectrum was created for each grating arm and diffraction order. To generate Swift spectra, the on-demand software was used, where the information of AE Aqr was manually registered [12, 24].

For the UV data, the level I event file (i.e. unscreened event data) of the Swift UVOT data was used. The events were screened using time-tagged quality information both internal and external to the file, by running the uvotscreen task with the event file as input file, and the orbit ephemeris file. A level II event data file (with corrected photon arrival times) was then created, from which a light curve necessary for pulse timing was generated. Then, an independent search for periodicities was performed using epoch folding method [17]. The light curve, in this case, was folded with large number of periods where 64 periods were searched with a phase bin of 16 per period, using the default epoch of the light curve of 13613 and the ephemeris of de Jager et al. (1994), and also a period resolution of of $2.98 \times 10^{-5} \mathrm{~s}$ which was an oversampling of the default Fourier period resolution.

\subsection{Results}

Figure 3 shows the background subtracted light curves from Chandra and Swift XRT data. These are predominantly soft, highly variable and dominated by flares. The gaps in the Swift data correspond to snapshot observations characteristic of a low-Earth orbit satellite. The pulse period obtained for each data set was $P_{\text {pulse }}=33.0767 \pm 0.0068 \mathrm{~s}$ and $33.0767 \pm 0.0003 \mathrm{~s}$ [24]. These are in line with the $33 \mathrm{~s}$ spin period of the white dwarf. The most probable UV pulse period, obtained by fitting a gaussian to the peak of the period-chi squared plot was $33.0767 \pm 0.0032 \mathrm{~s}$. Using the X-ray and UV pulse periods from Swift data, pulse profiles were plotted and compared (Figure 4). The X-ray pulse profile is characterized by small amplitude, single-peaked and broad sinusoidals, with the pulse maximum of the $33 \mathrm{~s}$ spin period of the white dwarf consistent with that obtained from the Suzaku data [29]. The UV pulse profile shows two peaks per cycle, consistent with ealier studies [10,11]. The two peaks imply emission from the two magnetic poles at (or close to) the surface of the white dwarf [8, 11].

Figure 5 shows the background subtracted spectra from Chandra and Swift XRT data, fitted with three-temperature vmekal models. These are characterised by emission lines, prominent in the lower energy band, i.e. $E_{\mathrm{X}} \leq 2 \mathrm{keV}$, implying emission from a uniform and optically region [24]. 

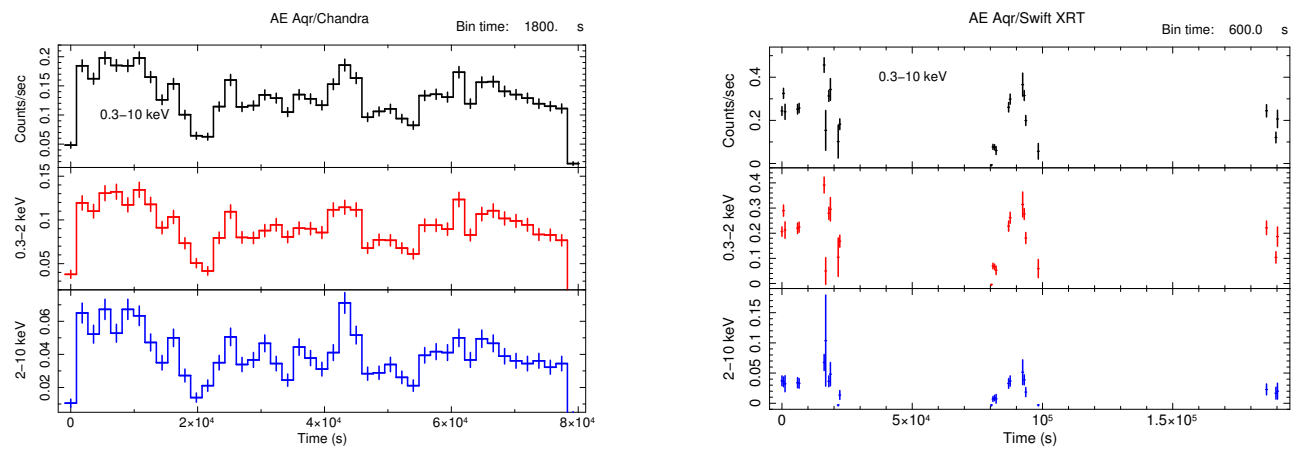

Figure 3. Background subtracted light curves of AE Aqr. Left: Chandra data. Right: Swift XRT data.
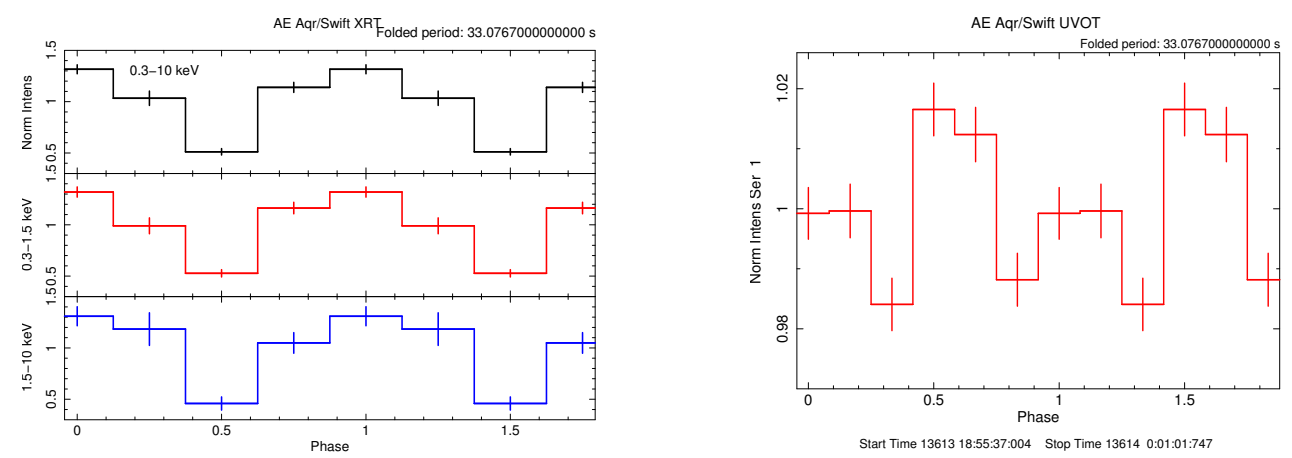

Figure 4. Pulse profiles of AE Aqr. Left: From XRT data Right: From UVOT data.

\section{Possible Emission Models}

Although the UV and X-ray luminosities of AE Aqr are the same $\left(L \sim 10^{31} \mathrm{erg} \mathrm{s}^{-1}\right)$, and also the pulse periods of the light curves are the same, the pulse profiles suggest that the UV and the X-ray emission are from different location within the system. The double-peaked profile of the UV light curve is associated with emission from the two magnetic poles of the white dwarf, possibly due to the drifting of material attached to the field lines at the magnetospheric radius. In this case, plasma instabilities at the Alfvén radius inhibits $100 \%$ magnetospheric propeller.

The source of soft X-ray emission in AE Aqr has been modeled to be the result of conversion of a fraction gravitational potential energy to heat energy of the ballistic stream at the Alfvén radius of the white dwarf [24]. The observed hard X-ray emission [29], has been modeled in terms of a pulsar-like process, in which particles are accelerated to relativistic energies, $\gamma>10^{7}$ [24]. This occurs in the regions where the synchrotron losses are less dominant compared to particle acceleration.

The spectral energy distribution (SED) of AE Aqr (constructed using catalogue, published and analysed data) is shown in Figure 6. The radio and far-IR data have been fitted with a power-law model, emission being attributed to synchrotron radiation of expanding magnetized blobs of relativistic electrons $[3,24]$. The near-IR and optical data are fitted with a thermal black body model of temperature of $T_{\mathrm{bb}, 1} \simeq 4.65 \times 10^{3} \mathrm{~K}$, and the emission is associated with the red star. The UV data fitted with another thermal black body model of temperature of $T_{\mathrm{bb}, 2} \simeq 10^{5} \mathrm{~K}$, originate from an emis- 

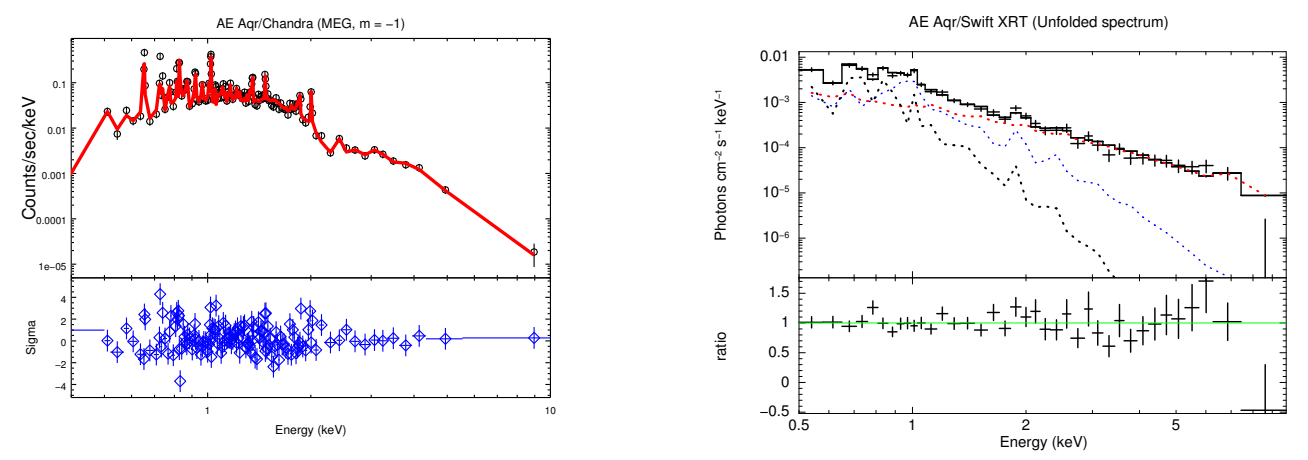

Figure 5. Background subtracted spectra of AE Aqr. Left: MEG spectrum for the diffraction order $m=-1$. Right: Unfolded spectrum from Swift XRT data.

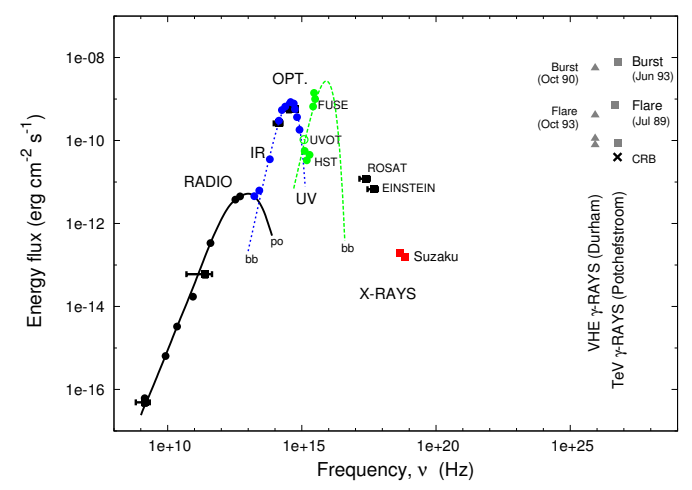

Figure 6. Spectral energy distribution of AE Aqr. Catalogue data are the black filled squares with errorbars, and the analysed data are the rest of the points. States of bursts and flares are indicated in the VHE and TeV $\gamma$-rays.

sion region of size $\sim 3 \times 10^{8} \mathrm{~cm}$, comparable with the radius of the white dwarf. The VHE and TeV $\gamma$-ray emission is most probably the result of burst-like particle acceleration events associated with the magnetospheric propeller process [20, 24].

\section{Summary}

The peculiar properties of AE Aquarii have been discussed within observational and theoretical framework. The multi-wavelength emission from AE Aqr is generally the result of a combination of processes. It has been shown that the X-ray emission is predominantly soft and characterised by flares and emission lines. The high variability and observed flares may suggest a very efficient magnetic propeller process and a state of turbulance at the propeller zone, i.e. sporadic mass accretion. The predominant soft X-ray emission with emission lines superimposed on the continuum suggest an optically thin emission plasma with uniform temperature distribution. The double-pulsed profile of the UV emission suggests emission from the two magnetic poles of the white dwarf. Highly relativistic electrons with $\gamma \sim 10^{7}$ may provide interesting possibility for high energy $\gamma$-ray production, and also 
exciting prospect for follow-up studies using Fermi and modern Cerenkov facilities like the High Energy Stereoscopic System (H.E.S.S.). This paper has made use of data supplied by the Chandra X-ray Center and UK Swift Science Data Centre. Busitema University provided the financial support.

\section{References}

[1] Abada-Simon M., Bastian T. S., Horne K., Bookbinder A. J., ASP Conf. Ser. 85, 355 (1995)

[2] Abada-Simon M., Lecacheux A., Bastian T.M., Bookbinder J. A., Dulk G.A., ApJ 406, 692 (1993)

[3] Bastian T. S., Dulk G. A., Chanmugam G., ApJ 324, 431 (1988)

[4] Bookbinder J. A., Lamb D. Q.,ApJ 323, 131 (1987)

[5] Bowden C. C. G., Bradbury S. M., Chadwick P. M., Dickinson et al., Astropart. Phys. 1, 47 (1992)

[6] Brissenden R. J., ASP Conf. Ser. 238, 22 (2001)

[7] Choi C-S., Dotani T., ApJ 646, 1149 (2006)

[8] Choi C-S., Dotani T., , Agrawal P. C., ApJ 525, 399 (1999)

[9] de Jager O. C., Meintjes P. J., O’Donoghue D., Robinson E. L., MNRAS 267, 577 (1994)

[10] Eracleous M., Horne K., Robinson E. L., et al., ApJ 433, 313 (1994)

[11] Eracleous M., Horne K., Osborne J. P., Clayton K. L., ASP Conf. Ser. 85, 392 (1995)

[12] Evans P. A., Beardmore A. P., Page K. L., Osborne J. P., et al., MNRAS 397, 1177 (2009)

[13] Gehrels N., Chincarini G., Giommi P., et al., ApJ 611, 1005 (2004)

[14] Ikhsanov N. K., A\&A 325, 1045 (1997)

[15] Ikhsanov N. R., Neustroev V. V., Beskrovnaya N. G., Astron. Letters 30, 675 (2004)

[16] Itoh K., Okada S., Ishida M., Kunieda H., ApJ 639, 397 (2006)

[17] Larsson S., A\&A Suppl. Ser. 117, 197 (1996)

[18] Mauche C. W., MNRAS 369, 1983 (2006)

[19] Mauche C. W., ApJ 706, 130 (2009)

[20] Meintjes P. J., de Jager O. C., MNRAS 311, 611 (2000)

[21] Meintjes P. J., de Jager O. C., Raubenheimer B. C., et al., ApJ 434, 292 (1994)

[22] Meintjes P.J., Raubenheimer B.C., de Jager O.C., et al., ApJ 401, 3251992

[23] Meintjes P. J., Venter L. A., MNRAS 360, 573 (2005)

[24] Oruru B., Meintjes P. J., MNRAS 421, 1557 (2012)

[25] Patterson J., ApJ 234, 978 (1979)

[26] Patterson J., Branch D., Chincarini G., Robinson E. L., ApJ 240, L133 (1980)

[27] Reinsch K., Beuermann K., Hanusch H., Thomas H. -C., ASP Conf. Ser. 85, 115 (1995)

[28] Schenker K., King A. R., Kolb U., Wynn G. A., Zhang Z.,MNRAS 337, 1105 (2002)

[29] Terada Y., Hayashi T., Ishida M., et al., Publ. Astron. Soc. Japan 60, 387 (2008)

[30] Venter L. A., Meintjes P. J., MNRAS 378, 681 (2007)

[31] Weisskopf M. C., Brinkman B., Canizares C., et al., Publ. Astron. Soc. Pacific 114, 1 (2002)

[32] Welsh W. F., Horne K., Oke J.B., ApJ 406, 229 (1993)

[33] Welsh W. F., Horne K., Gomer R., ApJ 410, L39 (1993)

[34] Welsh W. F., Horne K., Gomer R., MNRAS 275, 649 (1995)

[35] Wynn G. A., King R. A., MNRAS 275, 9 (1995)

[36] Wynn G. A., King R. A., Horne K., MNRAS 286, 436 (1997)

[37] Zinner E., Astron.Nach.265, 345 (1938) 